\title{
Proteolytic cleavage of elafin by 205 proteasome may contribute to inflammation in acute lung injury
}

\author{
Aoife Kerrin, ${ }^{1}$ Sinéad Weldon, ${ }^{1}$ Allen Hung-Kang Chung, ${ }^{2}$ Thelma Craig, ${ }^{1}$ \\ A John Simpson, ${ }^{3}$ Cecilia M O'Kane, ${ }^{1}$ Danny Francis McAuley, ${ }^{1}$ Clifford C Taggart $^{1}$
}

- Additional supplementary files are published online only. To view these files please visit the journal online (http://dx. doi.org/10.1136/thoraxinl2012-202536).

${ }^{1}$ Centre for Infection and Immunity, School of Medicine, Dentistry and Biomedical Sciences, Queen's University Belfast, Belfast, Northern Ireland, UK

${ }^{2}$ Laboratory of Biochemistry, National Heart, Lung, and Blood Institute, National Institutes of Health, Bethesda, Maryland, USA

${ }^{3}$ Institute of Cellular Medicine, University of Newcastle, Newcastle, UK

\section{Correspondence to} Professor Clifford Taggart, Centre for Infection and Immunity, School of Medicine, Dentistry and Biomedical Sciences, Queen's University Belfast, Health Sciences Building, 97 Lisburn Road, Belfast, Northern Ireland BT9 7BL, UK;

c.taggart@qub.ac.uk

AK and SW are joint first authors

Received 6 August 2012 Revised 14 November 2012 Accepted 22 November 2012 Published Online First 14 December 2012

\footnotetext{
To cite: Kerrin A, Weldon $S$, Chung $\mathrm{AH}-\mathrm{K}$, et al. Thorax 2013;68:315-321.
}

\begin{abstract}
Rationale We hypothesise that elafin levels in acute lung injury (ALI) decrease over time due, in part, to proteolytic degradation as observed in other lung diseases.

Objectives The aim of this study was to characterise temporal changes in elafin concentration in patients with $\mathrm{ALI}$ and to evaluate whether a decrease in elafin levels is due to elevated protease activity.

Methods Bronchoalveolar lavage fluid (BALF) was obtained from patients with ALI within $48 \mathrm{~h}$ of onset of ALI (day 0), at day 3 and at day 7. Elafin levels were quantified by ELISA. Elafin susceptibility to proteolytic cleavage by ALI BALF was assessed by Western blot and by high-performance liquid chromatography-mass spectrometry.

Measurements and main results Elafin levels were found to be significantly increased at the onset of ALI compared with healthy volunteers and fell significantly by day 7 compared with day 0 . In contrast, levels of secretory leukocyte protease inhibitor did not decrease over time. This decrease in elafin was due to cleavage by the 205 proteasome which was significantly increased in

\section{What is the key question?}

- Excessive inflammation is a hallmark feature of acute lung injury (ALI). This suggests that the host defence screen of the lung may be compromised during the inflammatory response

\section{What is the bottom line?}

- We have shown that the serine protease inhibitor, elafin, decreases in ALI over time due to proteolytic cleavage and inactivation by extracellular proteasome

\section{Why read on?}

- Elafin levels fall within the alveolar airspace as a direct consequence of proteolytic degradation by $20 \mathrm{~S}$ proteasome. Overall these findings suggest that a proteasome-mediated inactivation of an important lung host defence may contribute to the pathogenesis of $A L I$ and that antiprotease augmentation may be clinically beneficial.
\end{abstract} ALI BALF. Incubation of ALI BALF with the proteasome inhibitor epoxomicin confirmed that 20S proteasome protease activity was responsible for proteolytic cleavage of elafin, resulting in diminished anti-elastase activity. In addition, free neutrophil elastase activity significantly increased in ALI BALF from day 0 to day 7.

Conclusions Elafin concentrations fall within the pulmonary compartment over the course of $A L I$ as a result of proteolytic degradation. This loss of elafin may predispose people, in part, to excessive inflammation in ALI.

\section{INTRODUCTION}

Acute lung injury (ALI) is a major cause of morbidity and mortality in critically ill patients for which there is no effective pharmacological treatment. ${ }^{1}$ Elafin is a $6 \mathrm{kDa}$ serine protease inhibitor that has been isolated from lung secretions with concentrations in healthy lungs ranging from 1.5 to $4.5 \mu \mathrm{M}$ and is induced by pro-inflammatory mediators such as interleukin $1 \beta$, tumour necrosis factor $\alpha$, defensins, neutrophil elastase (NE) and lipopolysaccharide. ${ }^{2-6}$ Elafin is released by proteolytic cleavage from its precursor protein, trappin-2, to form the C-terminal 57-amino-acid mature elafin. ${ }^{7} 8$ The antiprotease activity of elafin resides within this C-terminal domain with specificity for NE and proteinase 3 and a transglutaminase substrate binding motif (GQDPVK) is present at the $\mathrm{N}$-terminus which allows it to cross-link extracellular matrix proteins. ${ }^{9}{ }^{10}$ In addition, elafin has antibacterial and immunomodulatory activities, which implicate it as an effector molecule in the host innate immune response. ${ }^{11-13}$

In the healthy lung, antiproteases are present at higher concentrations than their cognate proteases, thus providing the lung with a powerful antiinflammatory screen. In ALI, this protease-antiprotease balance is tipped in favour of proteases leading to dysregulated extracellular protease activity. ${ }^{14}$ Consequently, this protease burden can damage the alveolar epithelial-capillary endothelial barrier resulting in the production of pulmonary oedema within the alveolar space. ${ }^{15}$ As a result of inflammation and elevated protease activity, protective antiprotease levels may be compromised. A decrease in plasma elafin was found to correlate with altered elafin gene expression and was associated with increased acute respiratory distress syndrome (ARDS) risk. ${ }^{16-18}$

As a result it is important to evaluate elafin levels and activity in the lungs of patients with ALI as it has previously been demonstrated that elevated protease activity in certain lung diseases can degrade and inactivate host defence molecules such as elafin which subsequently affects their biological properties. $^{19-21}$ In this study, we investigated 
temporal levels of elafin in ALI bronchoalveolar lavage fluid (BALF) and correlated changes in levels to protease-mediated inflammation in ALI. We hypothesise that elafin levels in ALI decrease over time due, in part, to proteolytic degradation as observed in other lung diseases.

\section{MATERIALS AND METHODS Acquisition of BALF}

ALI BALF samples were acquired from the randomised clinical trial of Hydroxymethylglutaryl-Coenzyme A Reductase Inhibition for ALI as described previously. ${ }^{22}$ BALF from healthy volunteers and from intubated and mechanically ventilated patients 'at risk' of ALI as described previously ${ }^{23}$ were used as control groups. ALI patient aetiology is outlined in the online supplement.

\section{Determination of elafin, SLPI and proteasome levels in BALF}

Elafin, secretory leukocyte protease inhibitor (SLPI) and proteasome levels in BALF were determined by ELISA as previously described. ${ }^{19} 2425$

\section{Western blot analysis of recombinant elafin incubated with ALI BALF}

Recombinant human elafin (50 ng) was incubated with $10 \mu \mathrm{l}$ ALI BALF in tracheobronchial secretion in a final volume of $20 \mu \mathrm{l}$ for $24 \mathrm{~h}$ at $37^{\circ} \mathrm{C}$. In some experiments, ALI BALF was preincubated for $1 \mathrm{~h}$ at $37^{\circ} \mathrm{C}$ with a range of protease inhibitors, as indicated in the figure legends, before adding elafin. In other experiments, elafin and SLPI were incubated with $20 \mathrm{~S}$ proteasome for $10 \mathrm{~min}-24 \mathrm{~h}$ at $37^{\circ} \mathrm{C}$. Samples were separated by Tricine sodium dodecyl sulfate polyacrylamide gel electrophoresis (SDS-PAGE) on a $17.5 \%$ polyacrylamide gel and elafin and SLPI detected by Western blotting (see online supplement).

\section{S Proteasome and NE activity assay}

20S Proteasome and NE activity in BALF were measured using the fluorogenic substrates LLVY-aminomethylcoumarin (LLVY-AMC) and Ala-Ala-Pro-Val-aminomethylcoumarin (AAPV-AMC), respectively (see online supplement).

\section{HPLC mass spectrometry}

Cleavage of elafin by $20 \mathrm{~S}$ proteasome was assessed by incubating recombinant elafin $(500 \mathrm{ng})$ with recombinant $20 \mathrm{~S}$ proteasome $(5 \mu \mathrm{g})$ in $20 \mathrm{~S}$ buffer for $0-60 \mathrm{~min}$. Samples were then analysed by reverse-phase high-performance liquid chromatography (HPLC) coupled with electrospray mass spectrometry as previously described. ${ }^{19}{ }^{24}$ Experimental procedures are detailed in the online supplement.

\section{Evaluation of the anti-elastase activity of $20 \mathrm{~S}$ proteasome-cleaved elafin}

To evaluate the antiprotease activity of $20 \mathrm{~S}$ proteasome-cleaved elafin, we tested the ability of cleaved elafin to inhibit one of its target proteases, NE, using the substrate AAPV-AMC (see online supplement). Results are expressed as relative fluorescence units (RFUs).

\section{STATISTICAL ANALYSIS}

All data were analysed with GraphPad Prism V.5.0 (GraphPad Software Inc, San Diego, California, USA). Descriptive results of continuous variables were expressed as mean $( \pm$ SEM) or median (IQR) depending on the normality of their distribution. Unpaired t test or Mann-Whitney test were used as appropriate for comparison between two groups. For comparison between three or more groups, data were analysed by means of one-way analysis of variance or Kruskal-Wallis test using Dunn's multiple comparison test when significance was indicated (at the $p<0.05$ level). Statistical significance is presented as *p $<0.05, * \mathrm{p}<0.01$ and $* * \mathrm{p}<0.001$ in all figures.

\section{RESULTS}

\section{Levels of elafin and SLPI in ALI BALF}

As shown in figure 1A, BALF levels of elafin were significantly increased at the onset of ALI (30.66 ng/ml (16.61-56.01)) compared with healthy volunteers $(0.5 \mathrm{ng} / \mathrm{ml}(0.37-0.67))$. In addition, levels of elafin were significantly increased at the onset of ALI compared with the ventilated 'at risk' control group $(0.87 \mathrm{ng} / \mathrm{ml}(0.44-1.4))$. Levels of SLPI (figure $1 \mathrm{~B})$ were also significantly increased at the onset of ALI $(105 \mathrm{ng} / \mathrm{ml}(53.5-$ 224.4)) compared with the ventilated 'at risk' control group $(0.1 \mathrm{ng} / \mathrm{ml}(0.06-0.15))$ and healthy volunteers $(0.12 \mathrm{ng} / \mathrm{ml}$ $(0.08-0.15))$. Temporal measurements of elafin were assessed in patients with ALI at the onset of ALI (day 0), day 3 and day 7 of disease. As shown in figure 1C, levels of elafin fell significantly by day $3(12.73 \mathrm{ng} / \mathrm{ml}(6.89-39.31))$ and day 7 (10.34 ng/ml (5.74-30.64)) compared with day $0(30.66 \mathrm{ng} / \mathrm{ml}$ (16.61-56.01)). Unlike elafin, there was no significant change in the levels of SLPI by day $3(95.67 \mathrm{ng} / \mathrm{ml}(58.01-243.9))$ and day $7(97.12 \mathrm{ng} / \mathrm{ml}(70.94-189.2))$ compared with day 0 (105 ng/ml (53.5-224.4)) (figure 1D).

\section{Effects of ALI BALF incubation on recombinant elafin integrity}

Exogenous elafin was incubated with healthy control BALF and ALI BALF (figure 2). Elafin was resistant to proteolytic cleavage in healthy volunteer BALF with a band corresponding to intact elafin only detected in these samples (figure $2 \mathrm{~A}$ ). In contrast, exogenous elafin was susceptible to proteolytic cleavage in ALI BALF with the presence of an upper band representing intact elafin and a lower band corresponding to cleaved elafin fragments (figure 2B).

\section{Identification of protease family involved in cleavage of elafin in ALI BALF}

ALI BALF was preincubated with a range of non-specific protease inhibitors targeting a number of protease families for $1 \mathrm{~h}$ before adding recombinant elafin. After $24 \mathrm{~h}$ of incubation at $37^{\circ} \mathrm{C}$, samples were analysed by Western blot. As shown in figure $3 \mathrm{~A}$, preincubation with E64, leupeptin (cysteine protease inhibitors) and pepstatin (aspartic protease inhibitor) had no effect on elafin cleavage. In addition, preincubation with the specific matrix metalloproteinase (MMP) inhibitors (GM6001 and phosphoramidon) had no effect on elafin degradation, suggesting that an MMP is not involved in the proteolytic cleavage of elafin in ALI BALF. However, preincubation with the overlapping serine protease inhibitors, antipain and chymostatin, was found to successfully inhibit elafin degradation. Preincubation of ALI BALF with elastase inhibitors (SLPI, Alpha 1 Antitrypsin and MeOSuc-AlaAla-Pro-Val-chloromethylketone) did not inhibit degradation of elafin, suggesting that NE is not involved in ALI BALF-induced elafin cleavage (figure 3B). In addition, preincubation with anti-thrombin had no effect, suggesting that a number of the coagulation cascade proteases are not involved. Only the serine protease inhibitors TLCK and pefabloc successfully inhibited the proteolytic degradation of elafin, which indicated that a serine protease may be involved in the cleavage of elafin. 
Figure 1 Elafin and secretory leukocyte protease inhibitor (SLPI) levels are significantly increased in patients with acute lung injury (ALI) within the pulmonary compartment but only elafin levels fall over time. Elafin (A) and SLPI (B) levels were determined in bronchoalveolar lavage fluid (BALF) from healthy volunteers $(n=19)$, a ventilated 'at risk' control group $(n=12)$ and in patients with ALI $(n=37)$ by ELISA. * $p<0.05$, ${ }^{* *} p<0.01,{ }^{* * *} p<0.001$. Temporal measurements of elafin (C) and SLPI (D) in day $0(n=37)$, day $3(n=17)$ and day $7(n=9)$ ALI BALF were determined by ELISA. * $\mathrm{p}<0.05$ vs day 0 .
A

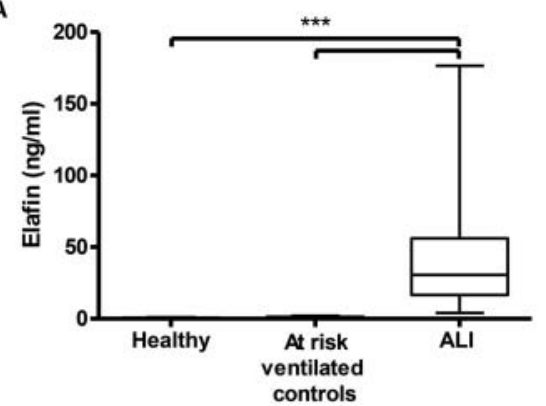

C

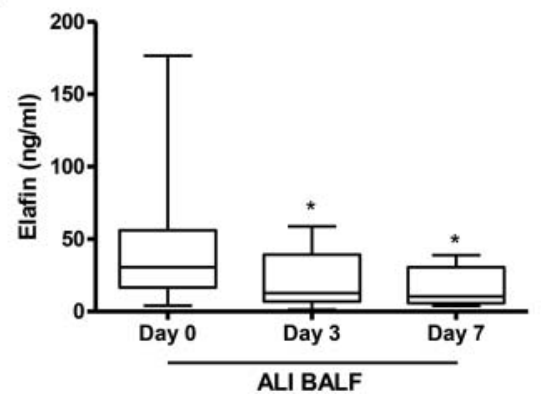

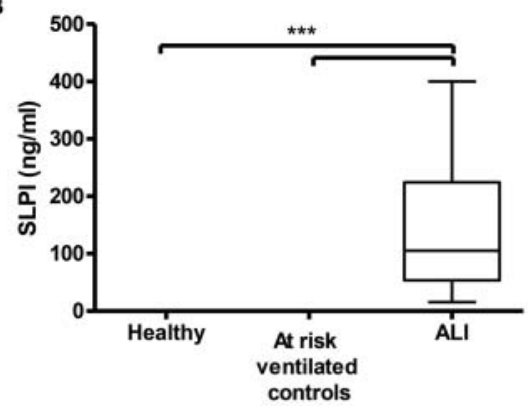

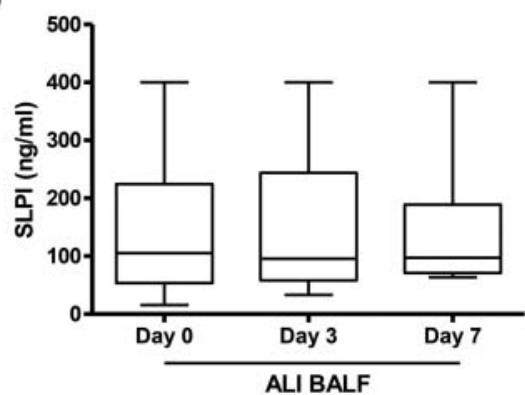

20S Proteasome is involved in mediating the proteolytic degradation of elafin

Overall, our findings indicated that a mixture of trypsin-like and chymotrypsin-like protease inhibitors abrogate the cleavage of elafin. The $20 \mathrm{~S}$ proteasome exhibits trypsin-like and chymotrypsin-like enzymatic activity and biologically active $20 \mathrm{~S}$ proteasome has been detected in the alveolar space of patients with ARDS and in the lungs in a rodent model of lung injury. ${ }^{25-27}$ To investigate if $20 \mathrm{~S}$ proteasome was responsible for the cleavage of elafin, we examined whether epoxomicin-a highly specific proteasome inhibitor-could inhibit cleavage of elafin. As shown in figure 4A, inhibition of elafin cleavage was observed with all concentrations of epoxomicin. A time-course incubation of $40 \mathrm{ng}$ exogenous elafin with $400 \mathrm{ng} 20 \mathrm{~S}$ proteasome was performed. As shown in figure $4 \mathrm{~B}$, incubation of recombinant elafin with active $20 \mathrm{~S}$ proteasome resulted in the appearance of elafin cleavage fragments, generated in a rapid time-dependent manner with cleavage of elafin occurring just after $30 \mathrm{~min}$ with full-length elafin degradation observed after $120 \mathrm{~min}$. In contrast, incubation of recombinant SLPI (100 ng) with $400 \mathrm{ng}$ of $20 \mathrm{~S}$ proteasome revealed only partial degradation of SLPI even after $24 \mathrm{~h}$ (figure 4C). Our results to this point suggest that $20 \mathrm{~S}$ proteasome is involved in the proteolytic degradation of elafin by ALI BALF as active 20S proteasome cleaved recombinant elafin in vitro. In addition, the specific proteasome inhibitor, epoxomicin, inhibited the cleavage of elafin.

\section{Measurement of 20S proteasome concentrations and activity in ALI BALF}

20S Proteasome concentrations from patients with ALI and healthy subjects were determined by ELISA. As shown in figure 5A, $20 \mathrm{~S}$ proteasome concentrations were significantly increased by day $0(342 \pm 79 \mathrm{ng} / \mathrm{ml})$ and day $3(271 \pm 110 \mathrm{ng} / \mathrm{ml})$ ALI BALF compared with healthy volunteer BALF (66 $2 \mathrm{ng} / \mathrm{ml})$. However, there was no significant difference between day 7 ALI BALF $(75 \pm 17 \mathrm{ng} / \mathrm{ml})$ and healthy BALF. 20S Proteasome activity levels in BALF from patients with ALI and healthy subjects were
Figure 2 Effects of healthy and acute lung injury (ALI) bronchoalveolar lavage fluid (BALF) incubation on recombinant elafin integrity. To investigate the effects of healthy and ALI BALF on the integrity of elafin, recombinant human elafin was incubated with healthy $(A)$ and $A L I$ BALF (B) at $37^{\circ} \mathrm{C}$ overnight. Samples were electrophoresed on a $17.5 \%$ Tricine/sodium dodecyl sulfate polyacrylamide gel electrophoresis (SDS-PAGE) under non-reducing conditions. Degradation of elafin was assessed by Western blot using a biotinylated anti-elafin antibody. Asterisks signify the variability in susceptibility to proteolytic degradation among patients. FL elafin, full-length elafin; C elafin, cleaved elafin.
A

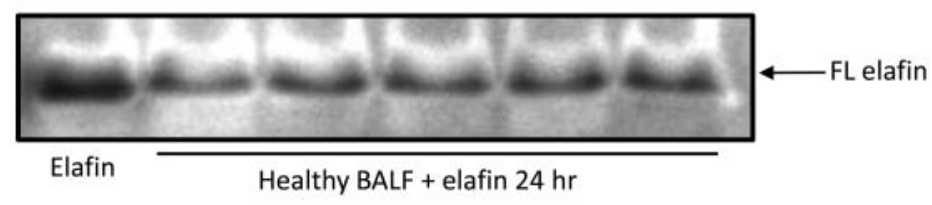

B

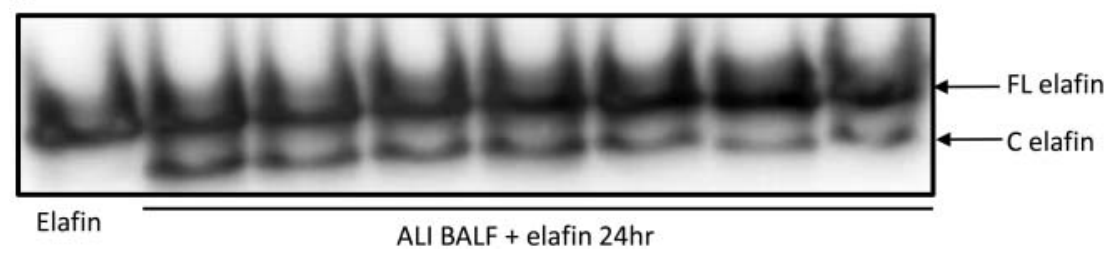


Figure 3 Identification of the protease(s) involved in the proteolytic cleavage of elafin by acute lung injury (ALI) bronchoalveolar lavage fluid (BALF). ALI BALF was preincubated for $1 \mathrm{~h}$ at $37^{\circ} \mathrm{C}$ with protease inhibitors directed towards various classes of protease (serine, aspartic, cysteine and metalloprotease) (A) or directed towards serine proteases only (B). Elafin was added to the samples and incubated for $24 \mathrm{~h}$ at $37^{\circ} \mathrm{C}$. Samples were electrophoresed on a $17.5 \%$ Tricine/sodium dodecyl sulfate polyacrylamide gel electrophoresis (SDS-PAGE) under non-reducing conditions and elafin detected by Western blot. FL elafin, full-length elafin; C elafin, cleaved elafin. SLPI, secretory leukocyte protease inhibitor.
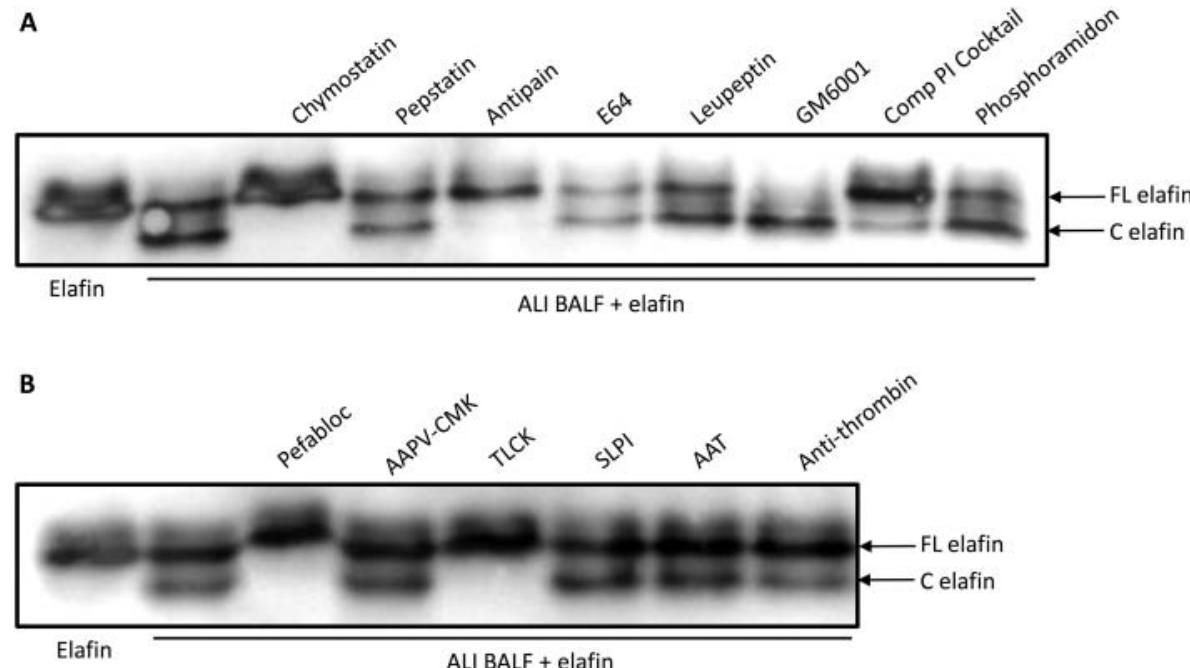

determined by cleavage of the fluorogenic substrate LLVY-AMC. As illustrated in figure $5 \mathrm{~B}, 20 \mathrm{~S}$ proteasome activity levels were significantly increased on day $0(19053 \pm 6777 \Delta$ RFU), day 3 $(9171 \pm 7980 \Delta$ RFU) and day 7 ALI BALF (4444 $\pm 2309 \Delta$ RFU) compared with healthy subjects $(97 \pm 73 \Delta \mathrm{RFU})$.

\section{Analysis of 20S proteasome-elafin cleavage sites}

Products from $20 \mathrm{~S}$ proteasome-elafin incubations were analysed by HPLC and mass spectrometry. As before, elafin (500 ng) was

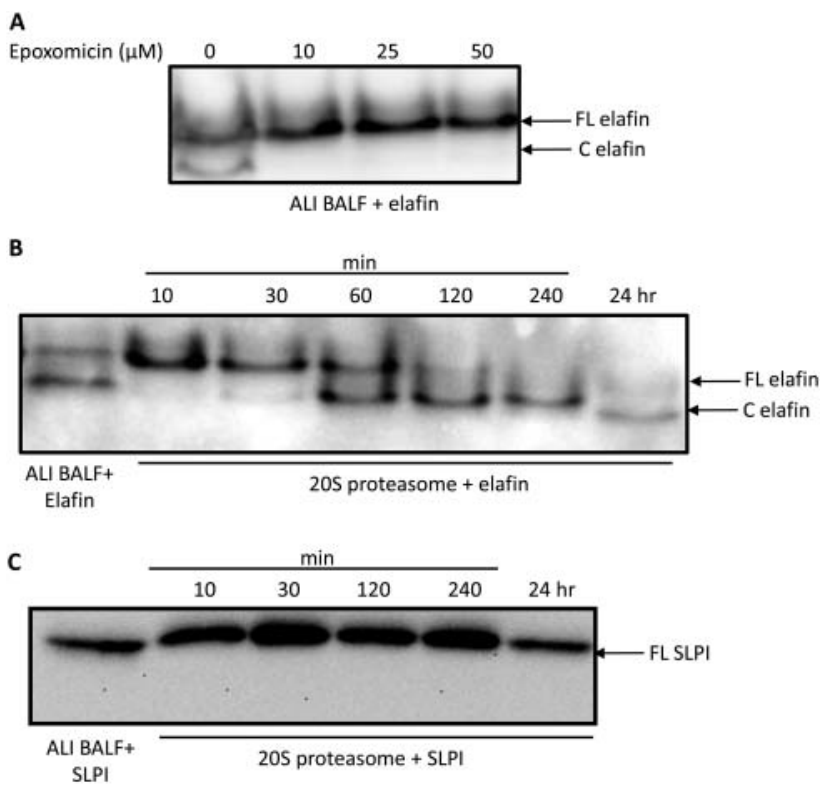

Figure 4 Effect of epoxomicin on acute lung injury (ALI) bronchoalveolar lavage fluid (BALF) induced cleavage of elafin. (A) ALI BALF was preincubated for $1 \mathrm{~h}$ at $37^{\circ} \mathrm{C}$ with the highly specific $20 \mathrm{~S}$ proteasome inhibitor epoxomicin $(10-50 \mu \mathrm{M})$ and incubated with elafin for $24 \mathrm{~h}$. (B, C) Time course incubation of active $20 \mathrm{~S}$ proteasome $(400 \mathrm{ng})$ with elafin $(40 \mathrm{ng})(\mathrm{B})$ and secretory leukocyte protease inhibitor (SLPI) (100 ng) (C) at various time points at $37^{\circ} \mathrm{C}$. Samples were electrophoresed on a $17.5 \%$ Tricine/sodium dodecyl sulfate polyacrylamide gel electrophoresis (SDS-PAGE) under non-reducing conditions and elafin and SLPI visualised by Western blot using a biotinylated anti-elafin antibody and biotinylated SLPI antibody, respectively. FL elafin, full-length elafin; $\mathrm{FL} \mathrm{SLPI,} \mathrm{full-length} \mathrm{SLPI;}$ C elafin, cleaved elafin. incubated with $20 \mathrm{~S}$ proteasome $(5 \mu \mathrm{g})$ for $0,15,30$ and $60 \mathrm{~min}$. One component of substantial area was detected in the elafin/20S proteasome samples at $15 \mathrm{~min}$ and later (data not shown) but not in the $0 \mathrm{~min}$ control (figure 6A). The measured monoisotopic mass was $2463.33 \mathrm{Da}$, in agreement with that calculated for the P1-24 fragment of elafin. Tandem mass spectrometric sequencing of the peptide established that this peptide fragment was P1-24, confirming that the proteasome attacks elafin by hydrolysing the peptide bond between $\mathrm{Ala}^{24}$ and $\mathrm{Met}^{25}$, as illustrated in figure 6B. Another elafin fragment (P1-23) was also detected in our samples, although this was present at lower levels than P1-24, thus suggesting that the primary cleavage site is at $\mathrm{Ala}^{24}-\mathrm{Met}^{25}$.

\section{Evaluation of the antiprotease activity of $20 \mathrm{~S}$ proteasome-cleaved elafin}

We evaluated the ability of $20 \mathrm{~S}$ proteasome-cleaved elafin to inhibit NE activity. As before, elafin (100 ng) was incubated with $20 \mathrm{~S}$ proteasome $(1 \mu \mathrm{g})$ and then incubated with a fixed amount of NE (500 ng). NE activity was measured using the NE substrate AAPV-AMC. As illustrated in figure 7A, 20S proteasome-cleaved elafin lost its ability to inhibit NE activity in contrast to intact elafin, which completely abolished NE activity. 20S Proteasome inactivated with epoxomicin prior to incubation with elafin did not inhibit elafin's anti-NE activity. Therefore, $20 \mathrm{~S}$ proteasome-cleaved elafin loses its antiprotease activity against its target serine protease NE. We confirmed that $20 \mathrm{~S}$ proteasome did not turn over the AAPV-AMC substrate (data not shown). We also evaluated free NE activity in ALI BALF. As shown in figure $7 \mathrm{~B}$, free $\mathrm{NE}$ activity significantly increased in ALI BALF on day 7 (590 \pm 319 RFU) compared with day 0 (92 $\pm 38 \mathrm{RFU})$, indicating that proteasome-mediated cleavage of elafin correlates to the decreased anti-NE screen in the ALI lung over time.

\section{DISCUSSION}

ALI is associated with a high mortality rate of up to $40 \%$. There is an urgent need to develop effective therapeutics for the treatment of this condition. ${ }^{28}$ The biological properties of elafin have been well described since its discovery, highlighting elafin as a potential therapeutic for the treatment of inflammatory lung conditions. ${ }^{11} 132930$ Due to the multi-factorial nature of ALI, which involves a complex network of pro-inflammatory 


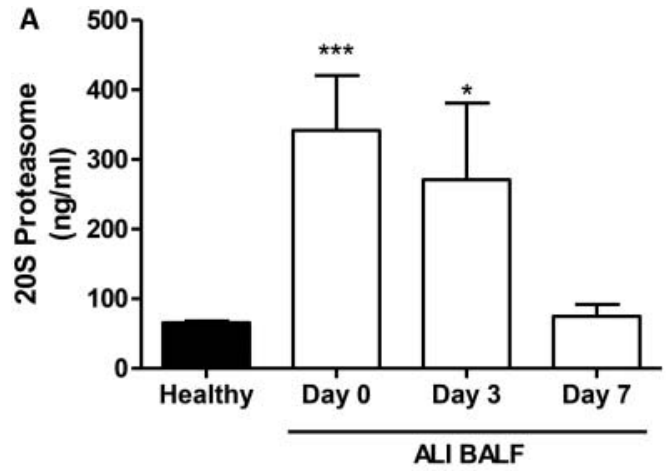

B

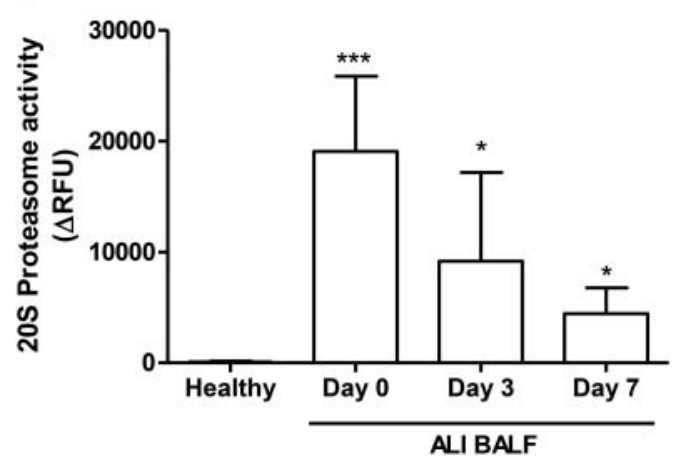

Figure 520 S Proteasome concentrations and activity levels in healthy volunteer and acute lung injury (ALI) bronchoalveolar lavage fluid (BALF). (A) 20S proteasome concentrations were measured in healthy and ALI BALF by ELISA. 20S Proteasome values are expressed as $\mathrm{ng} / \mathrm{ml}$. (B) 20S Proteasome activity in BALF from healthy volunteers and ALI BALF was measured using the fluorogenic substrate LLVY-AMC. The rate of substrate hydrolysis was monitored at $37^{\circ} \mathrm{C}$ over time and results were expressed as the change $(\Delta)$ in relative fluorescence units. 20 S Proteasome activity is expressed as actual 205 proteasome activity after correction for epoxomicin proteasome inhibiton. ${ }^{*} \mathrm{p}<0.05$ and ${ }^{* * *} p<0.001$ versus healthy BALF.

cytokines and protease activities, protective host defence proteins such as elafin may be left vulnerable to enzymatic cleavage and inactivation. In keeping with previous reports, we showed elafin and SLPI levels to be significantly increased in patients with ALI compared with healthy volunteers. ${ }^{31}$ To ensure that this increase in antiprotease levels observed at the onset of ALI was not induced by mechanical ventilation, we measured elafin and SLPI levels in BALF from intensive care unit patients who were mechanically ventilated but did not fulfil criteria for ALI. Elafin and SLPI levels within this 'at risk' control group were similar to those found in healthy volunteers, confirming that the increase in elafin levels at onset of ALI was not as a result of mechanical ventilation.

We extended these findings further by assessing the temporal measurements of anti-protease levels in ALI BALF over the course of 7 days. In contrast to SLPI, elafin levels fell significantly by day 3 and 7 compared with baseline ALI BALF (day $0)$. To our knowledge, these data are the first to demonstrate a significant decrease in the temporal levels of elafin within the pulmonary compartment in patients with ALI. Recent temporal measurements in whole blood samples from patients with ARDS revealed levels of plasma elafin which declined with the clinical progress of ARDS. ${ }^{18}$ This decrease in plasma elafin was found to correlate with downregulated elafin gene expression in the acute stage of ARDS. ${ }^{17} 18$ It is therefore plausible that the decreased levels of elafin over time observed in ALI BALF may, in part, be mediated by decreased elafin expression. However, we now demonstrate that elafin is susceptible to proteolytic cleavage in the ALI lung.

Recent research suggests that the $20 \mathrm{~S}$ proteasome is present and active in the extracellular space, although its function is as yet unclear. $^{32} 33$ Extracellular, biologically active $20 \mathrm{~S}$ proteasome has been detected in the alveolar space of patients with $\mathrm{ALI}^{25} 26$ and in a rodent model of lung injury. ${ }^{27}$ In addition, alveolar proteasome concentrations have been found to be markedly higher in patients with ALI, and in the circulation of patients with sepsis compared with healthy subjects. ${ }^{25} 34$ In agreement with previous data, ${ }^{25}$ we detected increased $20 \mathrm{~S}$ proteasome concentrations in BALF obtained at the onset of ALI (day 0) compared with healthy BALF samples. Temporal analysis revealed that the levels of BALF proteasome decreased over the course of ALI so that by day 7 there was no significant difference compared with healthy BALF. In contrast, we detected significantly increased $20 \mathrm{~S}$ proteasome activity levels in all ALI BALF compared with healthy subjects. However, similar to what was observed for concentrations, a temporal decrease in proteasome activity was also evident in ALI BALF. It is interesting to note the discrepancy between proteasome activity and levels in
Figure 6 High-performance liquid chromatography (HPLC) analysis of elafin incubated with 205 proteasome. (A) Recombinant elafin (500 ng) was incubated with recombinant $20 \mathrm{~S}$ proteasome $(5 \mu \mathrm{g})$ for 0 and $15 \mathrm{~min}$. Samples were analysed by reverse-phase HPLC coupled with electrospray mass spectrometry to identify the elafin fragments generated by $20 \mathrm{~S}$ proteasome cleavage. A major fragment representing elafin residues $\mathrm{P} 1-24$ and a minor cleavage product of P1-23 were detected. (B) Schematic representation of the primary $20 \mathrm{~S}$ proteasome cleavage site in the amino acid sequence of elafin ( Ala $^{24}-\mathrm{Met}^{25}$ ) indicated by the arrow, and the second minor cleavage site $\left(\mathrm{Cys}^{22}-\mathrm{Ala}^{23}\right)$ indicated by the asterisk.
A

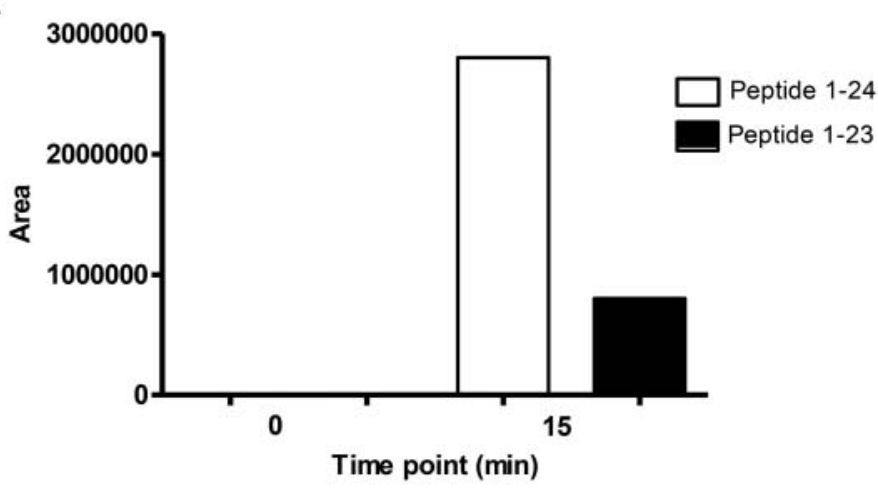

B

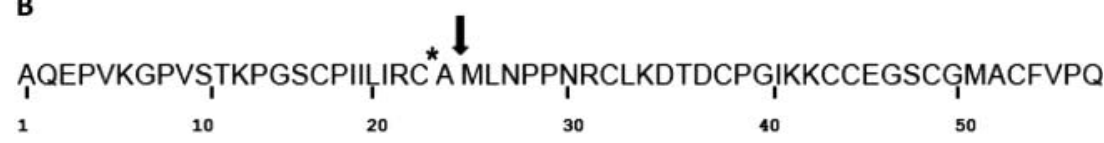


Figure 7 20S Proteasome-cleaved elafin loses its antiprotease activity and free neutrophil elastase (NE) activity increases in acute lung injury (ALI) bronchoalveolar lavage fluid (BALF). (A) 20S Proteasome-cleaved elafin loses its ability to inhibit activity of the serine protease NE. 20S proteasome inactivated with epoxomicin prior to addition of elafin retained elafin's anti-NE activity. NE activity was determined using the AAPV-AMC substrate and readings taken over time. Results are expressed as relative fluorescence units (RFUs).

(B) NE activity in BALF was measured using AAPV-AMC substrate. The rate of substrate hydrolysis was monitored at $37^{\circ} \mathrm{C}$ over time and the results were expressed as the change $(\Delta)$ in RFU. $\mathrm{NE}$ activity increased significantly in day 7 ALI BALF compared with day 0 BALF. ${ }^{*} \mathrm{p}<0.05$.
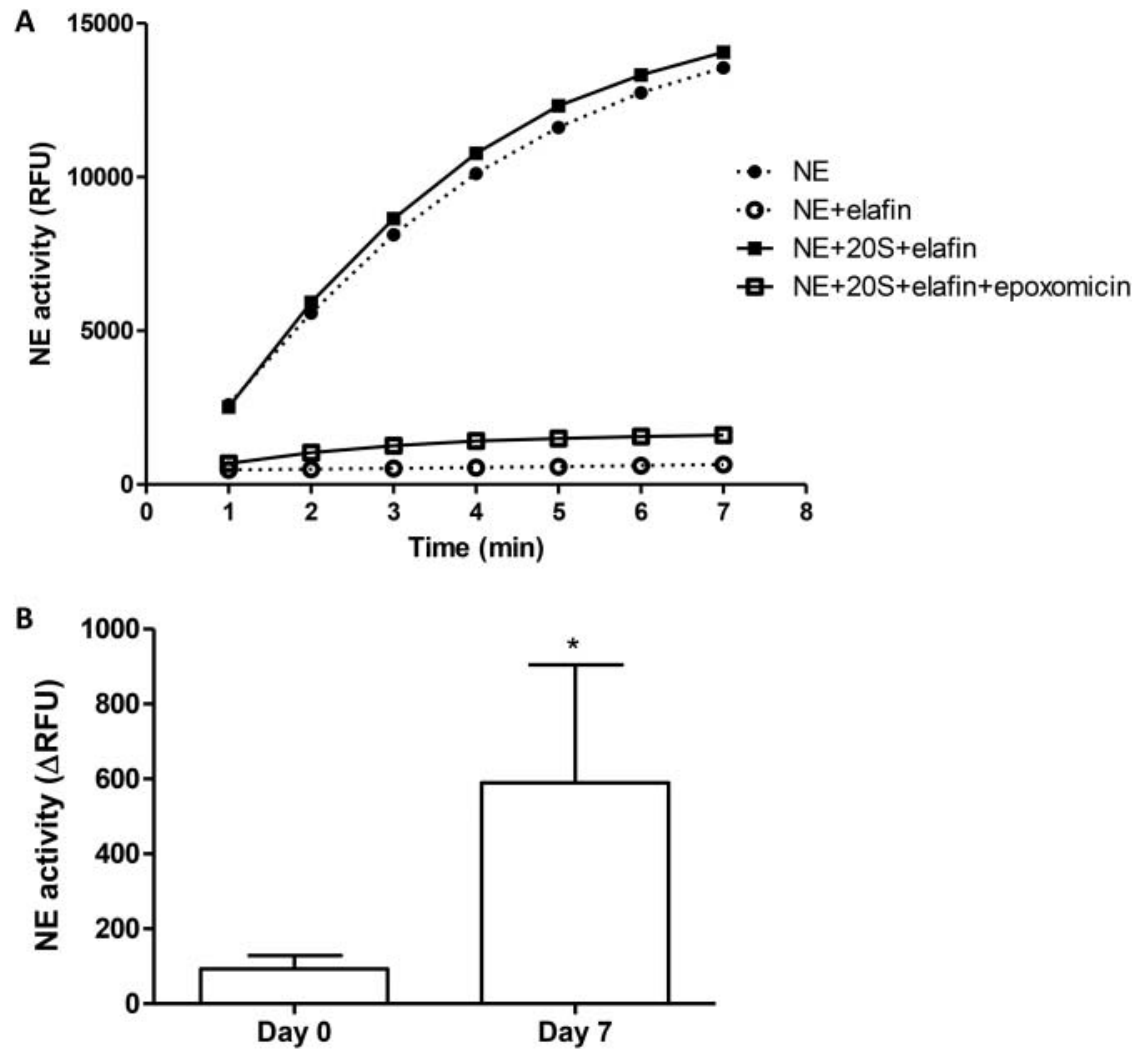

healthy and day 7 ALI BALF. Despite similar levels of proteasome in both sets of samples, there is significantly more proteasome activity in day 7 ALI BALF compared with healthy control samples. On the basis of our results and the limited evidence to date, it is possible that a proteasome inhibitor may be present in healthy BALF.

Our data conflict with a previous study which reported a 17-fold decrease of proteasomal activity detected in patients with severe ARDS compared with healthy subjects despite concentrations of proteasome being significantly higher in ARDS BALF compared with healthy control BALF. ${ }^{25}$ This decreased activity in patients with ARDS was suggested to be due to the presence of an unknown inhibitor present in the alveolar space. $^{25}$ To date, no extracellular proteasome inhibitors have been identified but the discovery of physiological inhibitors of the intracellular $20 \mathrm{~S}$ proteasome suggests that it is possible for extracellular proteasome inhibitors to be present in the lung. ${ }^{35}$ Regardless, the finding that preincubation of ALI BALF with epoxomicin-a specific proteasome inhibitor-abolished proteasome activity and inhibited ALI BALF-induced cleavage of elafin indicates $20 \mathrm{~S}$ proteasome activity is responsible for the proteolytic degradation of elafin. Further clarification of the role of $20 \mathrm{~S}$ proteasome in mediating ALI BALF-induced proteolytic cleavage of elafin was achieved by incubating exogenous elafin with active $20 \mathrm{~S}$ proteasome resulting in the appearance of elafin cleavage fragments generated in a time-dependent manner. In contrast to native, full-length elafin, these $20 \mathrm{~S}$ proteasomecleaved elafin fragments were unable to inhibit activity of NE. Pretreating ALI BALF with epoxomicin prevented the loss of elafin's anti-elastase activity. Interestingly, we demonstrated that NE activity in ALI BALF significantly increased from day 0 to day 7 , indicating that the proteasome cleavage of elafin may contribute in some part to increased NE activity in ALI. Free $\mathrm{NE}$ activity is well accepted as contributing directly to inflammation in ALI, resulting in microvascular injury, endothelial damage and interstitial oedema. ${ }^{36}$ To date, clinical trials utilising naturally occurring NE inhibitors have not been entirely successful due to problems with oxidative inactivation and proteolysis. ${ }^{36}$ However, elafin is currently in phase II trials for postoperative inflammation and may prove to be useful for treatment of ALI in future trials. ${ }^{37}$ It is possible that other mechanisms may contribute to this increased NE activity in day 7 ALI apart from proteasome-mediated elafin cleavage, including increased oxidation of other endogenous protease inhibitors such as $\alpha 1$ antitrypsin and SLPI or increased neutrophil necrosis. Further studies will be required to delineate fully the reasons for increased NE activity over time in the ALI lung.

In conclusion, this study shows elafin levels to decrease significantly over time in the pulmonary compartment of patients with ALI as a consequence of proteolytic degradation by the $20 \mathrm{~S}$ proteasome. This decrease may contribute, at least in part, to increased NE activity in ALI over time. Further research is warranted to investigate the cellular source of origin of the $20 \mathrm{~S}$ proteasome, its mechanism of transport into the extracellular space, regulation, expression, and above all, its biological role within the lung. The antiprotease deficiency as a result of cleavage by $20 \mathrm{~S}$ proteasome suggests that antiprotease augmentation in patients with ALI may be clinically beneficial in attenuating the excessive unregulated NE activity that persists in ALI. Elafin's multifunctional properties make it an attractive candidate as a therapeutic modality for ALI, although there is still a need for further research regarding its vulnerability to proteolytic degradation in vivo in conditions characterised by a protease burden such as ALI. 
Acknowledgements We would like to acknowledge helpful discussions with $\mathrm{Dr}$ Rod Levine (NHLBI, NIH, Bethesda, MD, USA) in the technical and written aspects of this study.

Contributors Conception, design, analysis, interpretation, drafting of manuscriptCCT, DFMCA, CMOK, SW, AK. Performed experiments-AK, AH-KC, CCT. Provided clinical samples-TC, DFMcA, AJS. All the authors reviewed the manuscript.

Funding Northern Ireland Chest Heart and Stroke Association (200941), HSC Research and Development, the Critical Care Translational Research Group, Critical Care Translational Research Group, Department of Education and Learning and Sir Jules Thorn Charitable Trust (AJS). This research was supported by the Intramural Research Program of the National Heart, Lung, and Blood Institute (AHKC).

\section{Competing interests None.}

Patient consent Obtained.

Provenance and peer review Not commissioned; internally peer reviewed.

\section{REFERENCES}

1 Rubenfeld GD, Caldwell E, Peabody E, et al. Incidence and outcomes of acute lung injury. N Engl J Med 2005;353:1685-93.

2 Tremblay GM, Sallenave JM, Israel-Assayag E, et al. Elafin/elastase-specific inhibitor in bronchoalveolar lavage of normal subjects and farmer's lung. Am J Respir Crit Care Med 1996:154(4 Pt 1):1092-8.

3 Sallenave JM, Shulmann J, Crossley J, et al. Regulation of secretory leukocyte proteinase inhibitor (SLPI) and elastase-specific inhibitor (ESI/elafin) in human airway epithelial cells by cytokines and neutrophilic enzymes. Am J Respir Cell Mol Biol 1994;11:733-41.

4 van Wetering S, van der Linden AC, van Sterkenburg MA, et al. Regulation of SLPI and elafin release from bronchial epithelial cells by neutrophil defensins. Am J Physiol Lung Cell Mol Physiol 2000;278:L51-8.

5 Reid PT, Marsden ME, Cunningham GA, et al. Human neutrophil elastase regulates the expression and secretion of elafin (elastase-specific inhibitor) in type II alveolar epithelial cells. FEBS Lett 1999:457:33-7.

6 Simpson AJ, Cunningham GA, Porteous DJ, et al. Regulation of adenovirus-mediated elafin transgene expression by bacterial lipopolysaccharide. Hum Gene Ther 2001;12:1395-406.

7 Tsunemi $\mathrm{M}$, Kato $\mathrm{H}$, Nishiuchi $\mathrm{Y}$, et al. Synthesis and structure-activity relationships of elafin, an elastase-specific inhibitor. Biochem Biophys Res Commun 1992;185:967-73.

8 Guyot N, Zani ML, Berger P, et al. Proteolytic susceptibility of the serine protease inhibitor trappin-2 (pre-elafin): evidence for tryptase-mediated generation of elafin. Biol Chem 2005;386:391-9

9 Molhuizen HO, Alkemade HA, Zeeuwen PL, et al. SKALP/elafin: an elastase inhibitor from cultured human keratinocytes. Purification, cDNA sequence, and evidence for transglutaminase cross-linking. J Biol Chem 1993:268:12028-32.

10 Nara $K$, Ito $S$, Ito $T$, et al. Elastase inhibitor elafin is a new type of proteinase inhibitor which has a transglutaminase-mediated anchoring sequence termed 'cementoin'. J Biochem 1994;115:441-8.

11 Simpson AJ, Maxwell Al, Govan JR, et al. Elafin (elastase-specific inhibitor) has anti-microbial activity against gram-positive and gram-negative respiratory pathogens. FEBS Lett 1999;452:309-13.

12 Sallenave JM, Cunningham GA, James RM, et al. Regulation of pulmonary and systemic bacterial lipopolysaccharide responses in transgenic mice expressing human elafin. Infect Immun 2003:71:3766-74.

13 Butler MW, Robertson I, Greene CM, et al. Elafin prevents lipopolysaccharide-induced AP-1 and NF-kappaB activation via an effect on the ubiquitin-proteasome pathway. J Biol Chem 2006;281:34730-35.

14 Petty TL. Protease mechanisms in the pathogenesis of acute lung injury. Ann N Y Acad Sci 1991;624:267-77.

15 Bachofen M, Weibel ER. Structural alterations of lung parenchyma in the adult respiratory distress syndrome. Clin Chest Med 1982;3:35-56.
16 Wang Z, Beach D, Su L, et al. A genome-wide expression analysis in blood identifies pre-elafin as a biomarker in ARDS. Am J Respir Cell Mol Biol 2008:38:724-32.

17 Wang Z, Chen F, Zhai $R$, et al. Plasma neutrophil elastase and elafin imbalance is associated with acute respiratory distress syndrome (ARDS) development. PLOS ONE 2009;4:e4380.

18 Tejera P, Wang Z, Zhai R, et al. Genetic polymorphisms of peptidase inhibitor 3 (elafin) are associated with ARDS. Am J Respir Cell Mol Biol 2009;41:696-704.

19 Guyot N, Butler MW, McNally P, et al. Elafin, an elastase-specific inhibitor, is cleaved by its cognate enzyme neutrophil elastase in sputum from individuals with cystic fibrosis. J Biol Chem 2008;283:32377-85.

20 Guyot N, Bergsson G, Butler MW, et al. Functional study of elafin cleaved by Pseudomonas aeruginosa metalloproteinases. Biol Chem 2010;391:705-16.

21 Kantyka T, Latendorf T, Wiedow 0 , et al. Elafin is specifically inactivated by RgpB from Porphyromonas gingivalis by distinct proteolytic cleavage. Biol Chem 2009;390:1313-20.

22 Craig TR, Duffy MJ, Shyamsundar M, et al. A randomized clinical trial of hydroxymethylglutaryl-CoA reductase inhibition for acute lung injury (The HARP study). Am J Respir Crit Care Med 2011;183:620-6.

23 Morris A, Kefala K, Wilkinson TS, et al. Diagnostic importance of pulmonary interleukin-1 $\beta$ and interleukin-8 in ventilator-associated pneumonia. Thorax 2010;65:201-7.

24 Weldon S, McNally P, McElvaney NG, et al. Decreased levels of secretory leucoprotease inhibitor in the Pseudomonas-infected cystic fibrosis lung are due to neutrophil elastase degradation. J Immunol 2009;183:8148-56.

25 Sixt SU, Adamzik M, Spyrka D, et al. Alveolar extracellular 20 S proteasome in patients with acute respiratory distress syndrome. Am J Respir Crit Care Med 2009;179:1098-106.

26 Sixt SU, Alami R, Hakenbeck J, et al. Distinct proteasome subpopulations in the alveolar space of patients with the acute respiratory distress syndrome. Mediators Inflamm 2012;2012:204250

27 Majetschak M, Sorell LT, Patricelli T, et al. Detection and possible role of proteasomes in the bronchoalveolar space of the injured lung. Physiol Res 2009:58:363-72.

28 Matthay MA, Zimmerman GA, Esmon C, et al. Future research directions in acute lung injury: summary of a National Heart, Lung, and Blood Institute working group. Am J Respir Crit Care Med 2003;167:1027-35.

29 Nobar SM, Zani ML, Boudier C, et al. Oxidized elafin and trappin poorly inhibit the elastolytic activity of neutrophil elastase and proteinase 3. FEBS J 2005;272:5883-93

30 Iqbal SM, Ball TB, Levinson P, et al. Elevated elafin/trappin-2 in the female genital tract is associated with protection against HIV acquisition. AIDS 2009:23:1669-77.

31 Sallenave JM, Donnelly SC, Grant IS, et al. Secretory leukocyte proteinase inhibito is preferentially increased in patients with acute respiratory distress syndrome. Eur Respir J 1999;13:1029-36

32 Sixt SU, Beiderlinden M, Jennissen HP, et al. Extracellular proteasome in the human alveolar space: a new housekeeping enzyme? Am J Physiol Lung Cell Mol Physiol 2007:292:L1280-8

33 Vadász I, Weiss $\mathrm{CH}$, Sznajder JI. Ubiquitination and proteolysis in acute lung injury. Chest 2012;141:763-71.

34 Roth GA, Moser B, Krenn C, et al. Heightened levels of circulating 20 S proteasome in critically ill patients. Eur I Clin Invest 2005;35:399-403.

35 Bao J, Sato K, Li M, et al. PR-39 and PR-11 peptides inhibit ischemia-reperfusion injury by blocking proteasome-mediated I kappa B alpha degradation. Am I Physiol Heart Circ Physiol 2001;281:H2612-8.

36 Korkmaz B, Horwitz MS, Jenne DE, et al. Neutrophil elastase, proteinase 3, and cathepsin $\mathrm{G}$ as therapeutic targets in human diseases. Pharmacol Rev 2010;62:726-59.

37 Shaw L, Wiedow 0. Therapeutic potential of human elafin. Biochem Soc Trans 2011:39:1450-4. 